$10 / 299395010$
SANDIA REPORT

SAND93-8503 - UC-401

Unlimited Release

Printed April 1993

RECEIVED

JUL 261993

Exchange Front Stability In

OSTI

Packed-Column Chromatography

S. K. Griffiths, R. H. Nilson

Propared by

Sandia National L sooratorias

Albuquerque, New Mexico 87185 and Livermore, Calliomin 94560

for tho Unived States Department of Energy

under Contract DE-ACO476DPOO7\% 
Issued by Sandia National Laboratorles, operated for the United States Department of Energy by Sandla Corporation.

NOTICE: This report was prepared as an account of work sponsored by an agency of the United States Government. Neither the United States Government nor any agency thereot, nor any of their employees, nor any of the contractors, subcontractors, or their employees, makes any war. ranty, express or implled, or assumes any legal llability or responsibility for the accuracy, completeness, or usefulness of any information, apparatus, product, or process disclosed, or represents that its use would not infringe privately owned rights. Reference herein to any specific commerclal product, process, or service by trade name, trademark, manufacturer, or otherwise, does not necessarily constitute or Imply its endorsement, recommendation, or favoring by the United States Government, any agency thereof or any of their contractors or subconractors. The views and opinions expressed herein do not necessarlly state or reflect those of the United States Government, any agency thereof or any of their contractors or subcontractors.

Thb report has bean reproduced from the best avallable copy.

Avalleble to DOE and DOE contractors from:

Offles of Sclentfic and Tochnical information

P.O. Box 62

Oak Fidoe TN 37831

Prloes avallabie from (615) 576-8401, FTs 6258401.

Avalleblo to the publle trom:

Natlonal Technled informetion Sorvice

U.S. Dopartment of Commarce

5285 Port Royal Rd.

Springfibld VA 22161.

NTIS prico codes

Printed copy: $\mathrm{AO}_{2}$

Mlerofichs copy: $\mathbf{A 0 1}$ 
SAND93-8503

UC-401

Printed April 1993

Unlimited Release

\title{
EXCHANGE FRONT STABILITY IN PACKED-COLUMN CHROMATOGRAPHY
}

\author{
Stewart K. Griffiths \\ Sandia National Laboratories \\ Livermore, California 94551-0969 \\ Robert H. Nilson \\ S-Cubed Division of Maxwell Labs \\ La Jolla, California 92038-1620
}

\begin{abstract}
Exchange front deformation in gas-phase chromatography may sometimes impair column performance. Such deformation can arise from column heterogeneities or frorn the unstable growth of small disturbances inherently present in the flow field. Here we examine the fundamental stability of a planar traveling exchange front subject to a small sinusoidal perturbation in the front position. The analysis includes the disparity between fluid and front velocities characteristic of exchange chromatography, as well as the distinction between fluid velocities ahead of and behind the front resulting from the net sorption of gases. Fluid compressibility is also considered. Disturbance growth rates are computed as a function of the wavelength, fluid viscosity ratio, and normalized front and fluid velocities over a wide range of each parameter. We find that growth rates increase with increasing viscosity ratio, decreasing wavelength, and increasing normalized front and fluid velocities. The conditions of neutral stability are also determined and indicate that fluid compressibility and net desorption tend to destabilize the exchange front. Sample calculations are presented for the problem of hydrogen isotope exchange in a column of palladium powder.
\end{abstract}

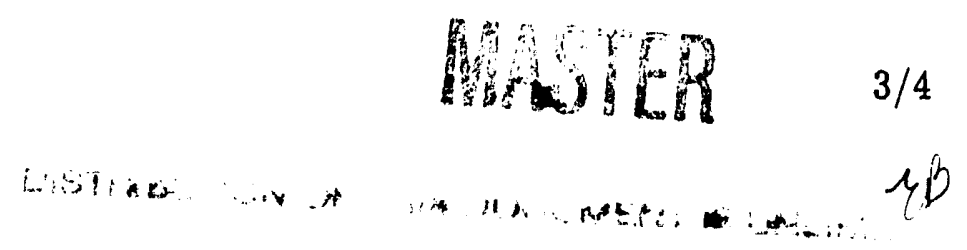


CONTENTS

Nomenclature . . . . . . . . . . . . . . . . . . . . 6 6

Introduction . . . . . . . . . . . . . . . . . . . . . . . . 7

Governing Equations . . . . . . . . . . . . . . . . . . . . . . . . . . . . . 8

Mathematical Method . . . . . . . . . . . . . . . . . . . . . . . . . 11

Time-Dependent Solutions . . . . . . . . . . . . . . . . . . . . . . . . . . 14

Disturbance Growth Rates and Neutral Stability . . . . . . . . . . . . . . . . 16

Sample Calculations . . . . . . . . . . . . . . . . . . . . . . . . . . . 21

Summary . . . . . . . . . . . . . . . . . . . . . . . . 25

References . . . . . . . . . . . . . . . . . . . 26 


\section{NOMENCLATURE}

$c_{p}$ fluid molar specific heat at constant pressure

$c_{v}$ solid molar specific heat at constant volume

$D_{i}$ fluid diffusivity $\left(D_{i}=\tilde{p} k / \epsilon \mu_{i}\right)$

$h$ species enthalpy of formation

$H$ total energy of fluid and solid

$k$ column permeability

$\ell \quad$ characteristic length $\left(\ell=\tilde{p} k / \mu_{1} \tilde{u}_{1}\right)$

$p \quad$ fluid pressure

$R \quad$ ideal gas constant

$t$ time

$T$ temperature

u fluid velocity

$u \quad x$ component of fluid velocity

$U$ front velocity

$v \quad y$ component of fluid velocity

$x \quad$ longitudinal spatial coordinate

$y$ transverse spatial coordinate

$z \quad$ exchange front position

$\alpha \quad$ disturbance spatial decay rate

$\beta$ disturbance wave number $(\beta=2 \pi / \lambda)$

$\epsilon \quad$ column porosity

$\lambda$ disturbance wavelength

$\mu \quad$ fluid viscosity

$\rho$ molar density

$\sigma$ disturbance temporal growth rate

$\phi \quad$ pressure gradient ratio $\left(\phi=\mu_{2} \tilde{u}_{2} / \mu_{1} \tilde{u}_{1}\right)$

$\psi$ influent stream dilution ratio $\left(\psi=\rho_{1, c} / \rho_{1, a}\right)$

Subscripts, Superscripts and Notation

1 behind exchange front

2 ahead of exchange front

$a$ active species in influent stream

$b$ active species initially on column

c carrier gas

$s \quad$ superscript denotes solid phase

* asterisk denotes normalized variable

1 prime denotes perturbation quantity

- overbar denotes mean flow quantity

- tilde denotes value at front location

- carat denotes perturbation constant 


\section{INTRODUCTION}

Packed-column saturation and elution chromatography are widely used in the drying, separation, purification, and identification of gases $[1,2]$. In all such applications species concentrations of the influent stream are altered by exchange with the porous solid, resulting in a wave-like concentration front traveling along the column. Because the front velocity is usually a small fraction of the mean fluid velocity, effluent gases are either enriched or depleted in selected species until breakthrough of the front at the column exit.

The efficiency of chromatographic processes generally depends on the sorption kinetics, equilibrium separation conditions, advective and diffusive transport near particle surfaces, and axial dispersion along the column [3]. In the case of porous or absorbing particles, the efficiency may also depend on intra-particle gas-phase or solid-phase diffusion [4]. When kinetic and species transport rates are large, the exchange front thickness is small, and the effluent stream shows an abrupt change in species concentration at breakthrough. Such conditions give high efficiency or utilization of the column. When rates are small the front thickness is large, and the effluent stream exhibits a gradual change in concentration as the front emerges from the column. These conditions give relatively poor column performance. In addition to these rate and transport phenomena, however, curvature of the exchange front may also affect column efficiency. The exchange process necessarily yields dissimilar fluid properties on either side of the front, and these may give rise to instabilities leading to large-scale front deformation $[5,6]$. If the exchange front radius of curvature falls to values comparable to the column diameter, the front will emerge from the column in a progressive fashion due solely to its geometry. This will again give a gradual change in breakthrough species concentrations and reduced column efficiency.

To help understand exchange front deformation we have analyzed the marginal stability of collocated temperature and species concentration jumps traveling in a porous medium. This analysis follows the classical approach used by Saffman and Taylor [7] to determine the rate of growth of a sinusoidal disturbance originating at the moving interface between two dissimilar fluids. The present problem, however, includes three considerations not previously addressed: (1) fluid compressibility; (2) a disparity between the front speed and the local fluid speeds; and (3) a disparity between the fluid speeds ahead of and behind the front. The first of these new considerations results in pressure disturbances that are not Laplacian, altering the relation between the transverse wavelength and longitudinal decay rate of the disturbance. The second consideration is specific to our exchange problem, and arises from the large ratio of solid-phase to gas-phase species concentrations found in some chromatography applications. The third consideration is peculiar to sorption problems in which total gas-phase continuity need not be satisfied across the front. All three considerations affect stability of the exchange front. 


\section{GOVERNING EQUATIONS}

We will consider a sharp exchange front traveling along a column, as shown in Figure 1. The front is initially planar, and its mean velocity $U$ is uniform over the column crosssection. Although the mean velocity is uniform, its value may vary with front position. With progress along the column, some portions of the front may travel faster or slower than the mean velocity, and this planar front will deform. Such deformation of the exchange front profile will disturb the initially uniform flow, leading to a two-dimensional and timedependent problem. Neglecting molecular diffusion, the species continuity equations for a compressible fluid are given by

$$
\frac{\partial}{\partial t}\left(\epsilon \rho_{i, j}\right)+\nabla \cdot\left(\rho_{i, j} \mathbf{u}_{i}\right)=0
$$

on each side of the front. Note that the continuity equations contain no sources or sinks as we allow sorptive processes only at the front location. The dependent variables $\rho_{i, j}$ and $\mathbf{u}_{i}$ are the partial molar density of species $j$ and the vector form of the apparent or superficial fluid velocity; $\epsilon$ is the column porosity, a parameter assumed to be constant in our analysis. Subscripts $i=1$ and 2 refer to the regions behind and ahead of the front, respectively, and the gradient operator is given by

$$
\nabla=\frac{\partial}{\partial x} \mathbf{i}+\frac{\partial}{\partial y} \mathbf{j}
$$

The independent variables $x$ and $y$ are the longitudinal and transverse spatial coordinates, $i$ and $\mathbf{j}$ are the associated unit direction vectors, and $t$ is time. Note that the continuity equations (1) also apply to the total molar densities in each of the two regions since these are simply the sums of all species contributions.

If fluid velocities are low, as is generally the case in packed-column flows, the momentum equations can be replaced with Darcy relations between the superficial fluid velocity and the local pressure gradient.

$$
\mathbf{u}_{i}=u_{i} \mathbf{i}+v_{i} \mathbf{j}=-\frac{k}{\mu_{i}} \nabla p_{i}
$$

Here $k$ is the column permeability, $\mu_{i}$ is the effective fluid viscosity, and $p_{i}$ is the total fluid pressure. The velocity components $u_{i}$ and $v_{i}$ are in the $x$ and $y$ directions, respectively. Further assuming that viscous dissipation is small, the energy equations in each region can be replaced with specified uniform temperatures. That is, temperatures in each of the two regions, $T_{1}$ and $T_{2}$, are assumed constant, but not necessarily equal. This still allows for a temperature jump in crossing the front.

Solutions to the transport equations must also satisfy matching conditions at the location of the moving exchange front. Without loss of generality we will consider only three distinct species: (1) one active species in the influent stream; (2) one active species initially on the column; and (3) an inert carrier gas. These are designated by the species subscripts $j=a, b$ 


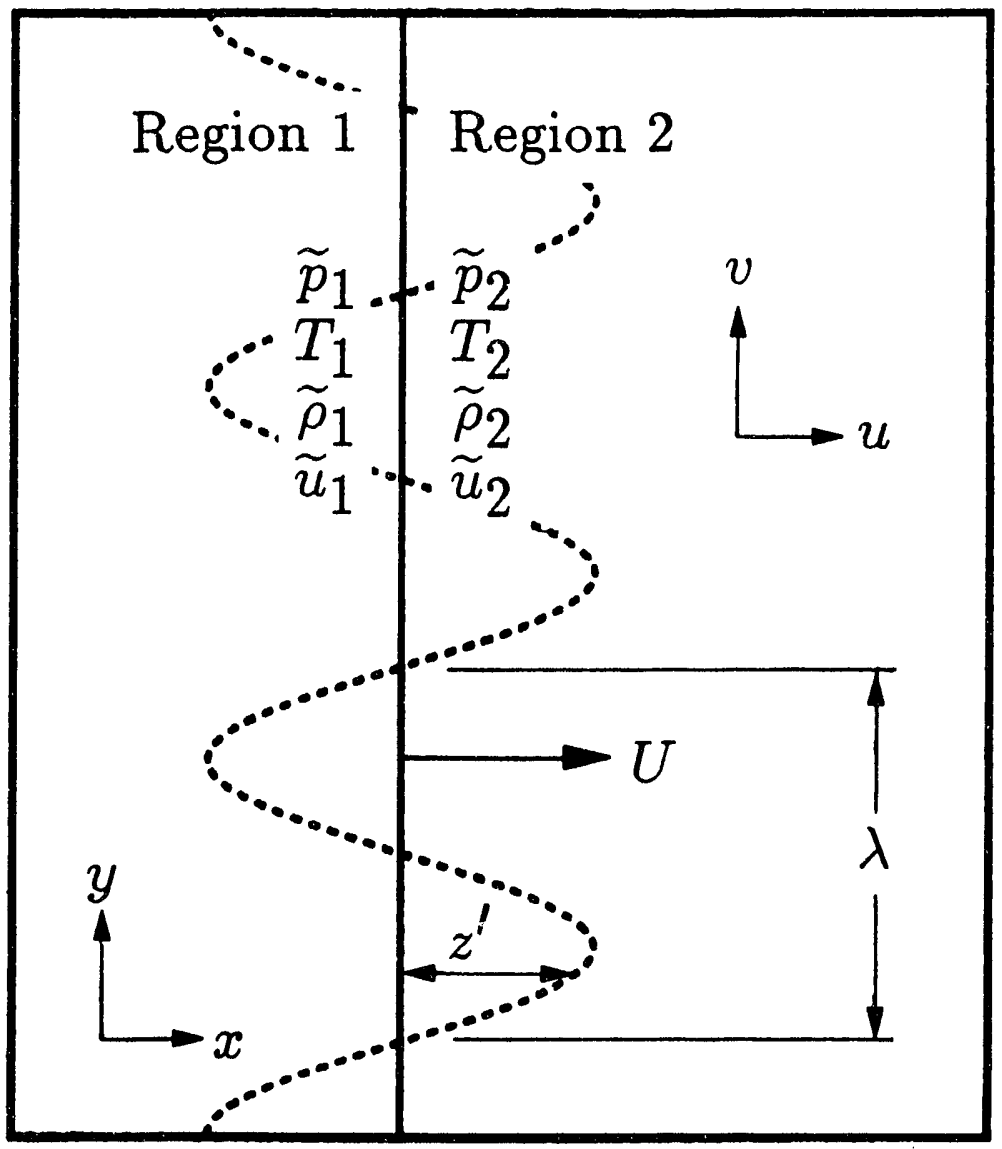

Figure 1. Schematic of the sharp moving exchange front. Species concentrations and temperatures are uniform on either side of the front but change abruptly at the front location. The fluid speeds $\tilde{u}_{1}$ and $\bar{u}_{2}$ are generally much larger than the velocity of the front and may differ from one another. Regions behind and ahead of the front are designated by the subscripts 1 and 2, respectively. 
and $c$, respectively. We further assume that the sorption or exchange in crossing the front is complete, such that species $a$ is present only in region 1 behind the front and the species $b$ is present only in region 2 ahead of the front. Under these restrictions the continuity matching conditions for the active species at a planar front are

$$
\rho_{i, j}\left(u_{i}-\epsilon U\right)=\rho_{i, j}^{s}(1-\epsilon) U \quad j=a, b
$$

where $\rho_{i, j}^{s}$ is the molar species density in the solici. Similarly, conservation of mass for the carrier gas requires that

$$
\rho_{1, c}\left(u_{1}-\epsilon U\right)=\rho_{2, c}\left(u_{2}-\epsilon U\right)
$$

Combining equations (4) and (5) the active species matching conditions can be rewritten in terms of the total densities $\rho_{1}$ and $\rho_{2}$,

$$
\rho_{i}\left(u_{i}-\epsilon U\right)=\rho_{i}^{\psi}(1-\epsilon) U
$$

in which $\rho_{1}^{\psi}=\rho_{1}^{s}(1+\psi)$ and $\rho_{2}^{\psi}=\psi \rho_{1}^{s}+\rho_{2}^{s}$ are the effective solid phase densities. The total fluid densities $\rho_{1}$ and $\rho_{2}$ in the two regions are given by the sum of the species densities, and $\psi=\rho_{1, c} / \rho_{1, a}$ is the dilution ratio of inert to active species in the influent stream. Note that large dilution ratios give small front velocities, as we would expect. Also note that $\rho_{1}^{s}=\rho_{1, a}^{s}$ and $\rho_{2}^{s}=\rho_{2, b}^{s}$ since each active species exists on only one side of the front.

Consistent with Darcy relations describing the fluid velocity, we neglect inertial contributions to the momentum balance at the exchange front. The momentum matching condition then requires simply that the pressure be continuous across the front; that is,

$$
p_{1}=p_{2}
$$

at the front location. This does not imply, however, that the pressure gradient also must be continuous.

Finally, the energy matching condition at the front requires that the net convective energy flux into the front is just balanced by the net source or sink associated with exchange between gas and solid phases,

$$
\rho_{1}\left(u_{1}-\epsilon U\right) c_{p, 1} T_{1}-\rho_{2}\left(u_{2}-\epsilon U\right) c_{p, 2} T_{2}=(1-\epsilon) U\left(H_{1}-H_{2}\right)
$$

where $H_{i}=\rho_{i}^{s}\left(h_{i}+c_{v, i}^{s} T_{i}\right)+\rho_{s} c_{v} T_{i}$ is the total internal and chemical energy of the solid phase constituents; $h_{i}$ is the molar enthalpy of formation of the active species in each region, $c_{p, i}$ is the effective molar specific heat of the fluid at constant pressure, $c_{v, i}^{s}$ is the specific heat of active species in the solid phase, and $c_{v}$ and $\rho_{s}$ are the specific heat and density of the solid. We have neglected conduction in equation ( $8 \mathrm{a})$, consistent with the sharp front approximation. Substituting the continuity matching conditions (6) into equation (8a) decouples the energy matching condition from the flow field, yielding an energy balance that is independent of both the fluid and front velocities.

$$
\rho_{1}^{\psi} c_{p, 1} T_{1}-\rho_{2}^{\psi} c_{p, 2} T_{2}=H_{1}-H_{2}
$$


Because the energy matching condition is independent of the flow, equation ( $8 \mathrm{a})$ or ( $8 \mathrm{~b})$ serves only to define the relation between the initial and final temperatures ahead of and behind the front, and the fluid motion can be computed without considering spatial variations in the temperature field, except for the jump at the front location.

The transport equations describing fluid motion are completed by an equation-of-state. We assume that the fluid is adequately described by an ideal gas relation, $p_{i}=\rho_{i} R T_{i}$, in which the total fluid densities $\rho_{1}$ and $\rho_{2}$ in the two regions are the sum of the partial molar densities of all species.

\section{MATHEMATICAL METHOD}

To evaluate exchange front stability we will perturb the front position by a small displacement $z^{\prime}$ and determine whether the initial displacement then grows or decays. This small disturbance is assumed to be periodic in the transverse direction and may either grow or decay exponentially in time. The local time-dependent front position in the moving coordinate system $z=x-U t$ is then given by

$$
z^{\prime}=\hat{z} e^{i \beta y} e^{\sigma t}
$$

where $\hat{z}$ is the initial amplitude of the front displacement, $\beta=2 \pi / \lambda$ is the displacement wavenumber, $\lambda$ is the corresponding wavelength, and $\sigma$ is the disturbance growth rate. The conditions of stable and unstable front deformation are determined by whether the unknown constant $\sigma$ is less than or greater than zero.

Deformation of the exchange front also perturbs the pressure, density and velocity fields. To account for this, each of the dependent variables is written as the sum of a steady undisturbed or mean value, denoted by overbar, and a time-dependent perturbation, denoted by a prime.

$$
\begin{gathered}
p_{i}=\bar{p}_{i}(x)+p_{i}^{\prime}(x, y, t) \quad \rho_{i}=\bar{\rho}_{i}(x)+\rho_{i}^{\prime}(x, y, t) \\
\mathbf{u}_{i}=\overline{\mathbf{u}}_{i}(x)+\mathbf{u}_{i}^{\prime}(x, y, t)
\end{gathered}
$$

In addition, the front velocity $U$ is written as the sum of the mean value $\bar{U}$ and the time derivative of the perturbed front position, $U=\bar{U}+U^{\prime}=\bar{U}+d z^{\prime} / d t$. These linear combinations are then substituted into the transport equations to yield a sei of equations governing the mean flow and time-dependent deviation from the mean.

For a uniform homogeneous column with constant pressures on both column ends, the mean flow is one-dimensional and the exchange front velocity is nearly constant. Only differences in the species compositions ahead of and behind the front can produce variations in the fluid and front velocities, and these variations depend only on the front position. 
As such, substituting (10b) and (10c) into the continuity equation (1) and grouping the inine-independent parts gives

$$
\nabla \cdot\left(\bar{\rho}_{i} \overline{\mathbf{u}}_{i}\right)=\frac{d}{d x}\left(\bar{\rho}_{i} \bar{u}_{i}\right)=0
$$

Similarly, substituting the total pressure (10a) into Darcy's relation (3) yields

$$
\bar{u}_{i}=-\frac{k}{\mu_{i}} \frac{d \bar{p}_{i}}{d x}
$$

Now substituting these results and the equation-of-state $\bar{p}_{i}=\bar{\rho}_{i} R T_{i}$ into the continuity equation (11) gives

$$
\frac{d}{d x}\left(\bar{p}_{i} \frac{d \bar{p}_{i}}{d x}\right)=0
$$

This is the familiar non-linear diffusion equation for compressible flow in a porous material. Again, the temperature in each region is assumed to be uniform and constant in time.

Matching conditions for the mean flow require that the steady parts of the mass flux and pressure satisfy the mass and momentum balances (6) and (7). Again replacing the pressures, densities and velocities with the linear combinations $(10 a, b, c)$ and collecting the steady parts yields

$$
\bar{\rho}_{i}\left(\bar{u}_{i}-\epsilon \bar{U}\right)=\rho_{i}^{\psi}(1-\epsilon) \bar{U}
$$

for the continuity equation, and

$$
\bar{p}_{1}=\bar{p}_{2}=\tilde{p}
$$

for conservation of momentum. A tilde over the pressure indicates its value at the front location. No new energy jump conditions are needed for the mean flow as temperatures on the two sides of the front must remain uniform and constant.

The pair of transport equations given by (13) possess a general solution of the parabolic form $\bar{p}_{i}^{2}=B_{i}-C_{i} x$, in which $B_{i}$ and $C_{i}$ are unknown constants. For a specified front position and initial column temperature $T_{2}$, these four unknown constants, the final temperature $T_{1}$, and the unknown front velocity $U$ can be calculated. The six governing algebraic equations are the two continuity matching conditions (14), the energy and momentum matching conditions ( $8 \mathrm{~b})$ and (15), and two pressure or flow rate boundary conditions at the column entrance and exit. The fluid densities $\bar{\rho}_{i}$ and velocities $\bar{u}_{i}$ required in the matching conditions can be computed using the general solution for the pressure and the equation-of-state or Darcy's relation (12). Solutions to the mean flow problem can be obtained readily by a simple iterative procedure.

Unlike the mean flow, the perturbed fluid motion is inherently two dimensional and time dependent. In addition, the transport equations for the time-dependent fluid motion contain products of the mean and perturbed quantities and therefore depend on the solutions to the 
mean flow problem. Again substituting (10b) and (10c) into the continuity equation and this time grouping the time-dependent parts yielis

$$
\epsilon \frac{\partial \rho_{i}^{\prime}}{\partial t}+\nabla \cdot\left(\bar{\rho}_{i} \mathbf{u}_{i}^{\prime}+\rho_{i}^{\prime} \overline{\mathbf{u}}_{i}\right)=0
$$

Note that we have assumed here that the column porosity is constant and that the higher order term $\nabla \cdot\left(\rho_{i}^{\prime} \mathbf{u}_{i}^{\prime}\right)$ can be neglected. The Darcy relations for the time-dependent fluid velocities are

$$
u_{i}^{\prime}=-\frac{k}{\mu_{i}} \frac{\partial p_{i}^{\prime}}{\partial x} \quad v_{i}^{\prime}=-\frac{k}{\mu_{i}} \frac{\partial p_{i}^{\prime}}{\partial y}
$$

and the equation-of-state for the perturbed variables is $p_{i}^{\prime}=\rho_{i}^{\prime} R T_{i}$. Substituting these expressions into the time-dependent continuity equation (16) gives a pair of transient linear diffusion equations for the perturbed fluid pressures.

$$
\frac{\epsilon \mu_{i}}{\bar{p}_{i} k} \frac{\partial p_{i}^{\prime}}{\partial t}=\frac{\partial^{2} p_{i}^{\prime}}{\partial x^{2}}+\frac{\partial^{2} p_{i}^{\prime}}{\partial y^{2}}+\frac{2}{\bar{p}_{i}} \frac{d \bar{p}_{i}}{d x} \frac{\partial p_{i}^{\prime}}{\partial x}+\frac{p_{i}^{\prime}}{\bar{p}_{i}} \frac{d^{2} \bar{p}_{i}}{d x^{2}}
$$

Since the perturbations of interest originate at the exchange front, we can replace the mean pressures and mean pressure derivatives with their local values. From equations (12), (13) and (15) these may be expressed as

$$
\bar{p}_{i}=\bar{p} \quad \frac{d \bar{p}_{i}}{d x}=-\frac{\mu_{i} \tilde{u}_{i}}{k} \quad \frac{d^{2} \bar{p}_{i}}{d x^{2}}=-\frac{1}{\tilde{p}}\left(\frac{\mu_{i} \bar{u}_{i}}{k}\right)^{2}
$$

where $\tilde{u}_{i}$ designates the $x$ component of the mean fluid velocity immediately adjacent to the exchange front. Making these substitutions in equation (18) and transforming the resulting expression into the moving spatial coordinate $z=x-\bar{U} t$ gives

$$
\frac{\partial p_{i}^{\prime}}{\partial t}=D_{i}\left(\frac{\partial^{2} p_{i}^{\prime}}{\partial z^{2}}+\frac{\partial^{2} p_{i}^{\prime}}{\partial y^{2}}\right)+\left(\bar{U}-2 \frac{\tilde{u}_{i}}{\epsilon}\right) \frac{\partial p_{i}^{\prime}}{J z}-\frac{1}{D_{i}}\left(\frac{\tilde{u}_{i}}{\epsilon}\right)^{2} p_{i}^{\prime}
$$

in which $D_{i}=\tilde{p} k / \epsilon \mu_{i}$ is the apparent diffusivity of a gas in a permeable porous medium. Note that the term $\bar{u}_{i} / \epsilon$ is the interstitial fluid speed.

The perturbed fluid motion must also satisfy matching conditions on the exchange front. In this case, however, the front is continuously deforming, and the matching conditions are time dependent. Conservation of mass requires that the net flux into or out of the front be zero; using equation (14) taking into account that $d\left(\bar{\rho}_{i} \bar{u}_{i}\right) / d z=0$, this may be stated as

$$
\rho_{i}^{\prime}\left(\tilde{u}_{i}-\epsilon \bar{U}\right)+\tilde{\rho}_{i}\left(u_{i}^{\prime}-\epsilon U^{\prime}\right)-\epsilon \bar{U} z^{\prime} \frac{d \bar{\rho}_{i}}{d z}=\rho_{i}^{\psi}(1-\epsilon) U^{\prime}
$$

Conservation of momentum for the perturbed flow requires that the time-dependent pressure be continuous across the deformed front, or

$$
p_{1}^{\prime}+z^{\prime} \frac{d \bar{p}_{1}}{d z}=p_{2}^{\prime}+z^{\prime} \frac{d \bar{p}_{2}}{d z}
$$


at the deformed front location. Note that the spatial derivatives of the mean density and pressure in equations (21) and (22) are advective perturbations. They are due to the timedependent displacement $z^{\prime}$ of the exchange front in the non-uriform steady fields ahead of and behind the front. Again, there are no distinct energy matching conditions for the timedependent problem because the temperatures are still specified as $T_{1}$ and $T_{2}$ on the two sides of the now deformed front. Had we instead assumed that these temperature fields also were perturbed by front deformation, the result here would be simply $T_{1}^{\prime}=T_{2}^{\prime}=0$ since the energy matching condition ( $8 \mathrm{~b})$ contains no other time-dependent quantities.

\section{TIME-DEPENDENT SOLUTIONS}

To solve the time-dependent transport equations and matching conditions we will introduce candidate solutions in a form that satisfies the governing differential equation (20) as well as the equation-of-state and subsidiary equations (17a) and (17b) relating the fluid velocity to the pressure gradients. The candidate solutions for the six dependent variables are

$$
\begin{gathered}
p_{i}^{\prime}=\hat{p}_{i} e^{i \beta y} e^{ \pm \alpha_{i} z} e^{\sigma t} \quad \rho_{i}^{\prime}=\frac{\hat{p}_{i}}{R T_{i}} e^{i \beta y} e^{ \pm \alpha_{i} z} e^{\sigma t} \\
u_{i}^{\prime}=\mp \frac{\hat{p}_{i} k}{\mu_{i}} \alpha_{i} e^{i \beta y} e^{ \pm \alpha_{i} z} e^{\sigma t}
\end{gathered}
$$

where $\hat{p}_{i}$ are constants, as are the exponential factors $\alpha_{i}, \beta$ and $\sigma$. These exponential solutions are compatible with the spatial periodicity and time variation of the front deformation $z^{\prime}$, and further satisfy the requirement that a disturbance originating at the front should decay with dis'ance from the mean front position. In order that the spatial decay constants $\alpha_{1}$ and $\alpha_{2}$ both be non-negative, equations $(23 a, b, c)$ are written with upper and lower signs referring to the regions behinc and ahead of the front, respectively.

Substituting the candidate solutions $(23 a, b, c)$ into the governing equations for the pressure $(20)$ yields a pair of algebraic relations between the exponential constants and the local parameters $\tilde{u}_{i}$ and $\bar{U}$ of the mean flow.

$$
\sigma=D_{i}\left(\alpha_{i}^{2}-\beta^{2}\right) \pm\left(\bar{U}-2 \frac{\bar{u}_{i}}{\epsilon}\right) \alpha_{i}-\frac{1}{D_{i}}\left(\frac{\tilde{u}_{i}}{\epsilon}\right)^{2}
$$

Similarlv, the front mass balances (21) reduce to the form

$$
\frac{\hat{p}_{i}}{\hat{z}}\left(\bar{u}_{i}-\epsilon \bar{U} \mp \epsilon \alpha_{i} D_{i}\right)=\left[\epsilon \bar{p}+(1-\epsilon) \rho_{i}^{\psi} R T_{i}\right] \sigma-\frac{\bar{U}}{D_{i}} \tilde{p} \tilde{u}_{i}
$$

in which the density gradient has been written in terms of the local velocity $\tilde{u}_{i}$ of the mean flow. Making the same substitutions in the pressure matching condition (22) gives

$$
\frac{\bar{p}}{\epsilon}\left(\frac{\tilde{u}_{2}}{D_{2}}-\frac{\tilde{u}_{1}}{D_{1}}\right)=\frac{\hat{p}_{2}-\hat{p}_{1}}{\bar{z}}
$$


Equations (24) through (26) form a system of five equations in six unknowns. However, the unknowns $\widehat{p}_{1}, \hat{p}_{2}$ and $\widehat{z}$ appear only in the ratio $\hat{p}_{i} / \widehat{z}$. Because of this, $\hat{p}_{i}$ and $\bar{z}$ may be eliminated by combining equations (25) and (26), to obtain a system of three equations in three unknowns.

To reduce our results to a relatively compact form, we will introduce a set of dimensionless parameters and dependent variables. The dimensionless variables are obtained through scaling the original values by an internal length $\ell$ and time $\ell \ell / \tilde{u}_{1}$,

$$
\beta^{*}=\frac{2 \pi}{\lambda^{*}}=\beta \ell \quad \alpha_{i}^{*}=\alpha_{i} \ell \quad \sigma^{*}=\sigma \frac{\epsilon \ell}{\tilde{u}_{1}}
$$

where the characteriatic length based on Darcy's relation is

$$
\ell=\frac{\tilde{p} k}{\mu_{1} \bar{u}_{1}}=\frac{\epsilon D_{1}}{\bar{u}_{1}}
$$

The corresponding parameters $U^{*}=\epsilon \bar{U} / \bar{u}_{1}, u_{2}^{*}=\tilde{u}_{2} / \bar{u}_{1}$ and $\mu_{2}^{*}=\mu_{2} / \mu_{1}$ are obtained by scaling the front velocity, fluid velocity and viscosity by the mean fluid speed $\tilde{u}_{1}$ and viscosity $\mu_{1}$ just behind the exchange front. With this change of variables equations (24) can be rewritten as

$$
\begin{gathered}
\alpha_{1}^{* 2}-\left(2-U^{*}\right) \alpha_{1}^{*}=\beta^{* 2}+\sigma^{*}+1 \\
\alpha_{2}^{* 2}+\mu_{2}^{*}\left(2 u_{2}^{*}-U^{*}\right) \alpha_{2}^{*}=\beta^{* 2}+\mu_{2}^{*} \sigma^{*}+\left(\mu_{2}^{*} u_{2}^{*}\right)^{2}
\end{gathered}
$$

and equations (14), (25) and (26) combine to yield

$$
\left(\mu_{2}^{*} u_{2}^{*}-1\right) A_{1} A_{2}+U^{*}\left(\mu_{2}^{* 2} u_{2}^{*} A_{1}+A_{2}\right)=\frac{\sigma^{*}}{U^{*}}\left(\mu_{2}^{*} u_{2}^{*} A_{1}+A_{2}\right)
$$

in which $A_{1}=\alpha_{1}^{*}-\left(1-U^{*}\right)$ and $A_{2}=\alpha_{2}^{*}+\mu_{2}^{*}\left(u_{2}^{*}-U^{*}\right)$. In the case of incompressible flow, equations (29) and (30) degenerate to $\alpha_{1}^{*}=\alpha_{2}^{*}=\beta^{*}$, and the corresponding form of equation (31) is

$$
\left(\mu_{2}^{*} u_{2}^{*}-1\right) \alpha_{1}^{*} \alpha_{2}^{*}=\frac{\sigma^{*}}{U^{*}}\left(\mu_{2}^{*} u_{2}^{*} \alpha_{1}^{*}+\alpha_{2}^{*}\right)
$$

These results cannot be obtained, however, directly from equation (31) by simpie parameter substitutions. Because density gradients in an ideal gas are proportional to gradients in the pressure, and these are equivalent to fluid velocities, equation (31) was obtained without an additional parameter to account for compressibility. Accordingly, equation (32) can be derived only by setting $\rho_{i}^{\prime}=0$ and $d \bar{\rho}_{i} / d z=0$ in equations (16) and (21) and dropping all subsequent contributions from these terms. Further setting $u_{2}^{*}=U^{*}=1$ in equation (32) yields the familiar Saffman-Taylor expression for the stability of a displacement front in a porous material or Hele-Shaw cell [7]. 


\section{DISTURBANCE GROWTH RATES AND NEUTRAL STABILITY}

The algebraic equations (29), (30) and (31) can be solved simultaneously for the three unknowns $\alpha_{1}^{*}, \alpha_{2}^{*}$ and $\sigma^{*}$ in terms of the four parameters $u_{2}^{*}, \mu_{2}^{*}, U^{*}$ and $\beta^{*}$. For this we use an iterative technique employing the ZEROIN [8] root finder. Given values for the four parameters, the procedure is to first guess a value of $\sigma^{*}$. Using this guessed value, the two quadratic equations (29) and (30) are solved directly for $\alpha_{1}^{*}$ and $\alpha_{2}^{*}$. Each equation possesses only one real positive root. These tentative values of $\alpha_{i}^{*}$ and $\sigma^{*}$ are then used in equation (31) to compute a residual for ZEROIN. The ZEROIN routine then selects a new value for $\beta^{*}$, and this process is repeated until the relative or absolute error in $\beta^{*}$ is reduced to $10^{-4}$.

Disturbance growth rates at a normalized fluid velocity $u_{2}^{*}=1.0$ and front velocity $U^{*}=1.0$ are shown in Figure 2 as a function of the normalized wavelength $\lambda^{*}$ for various values of the viscosity ratio $\mu_{2}^{*}$. We see that the viscosity ratio strongly affects both the growth rate magnitude and its sign. In addition, for some values of the viscosity ratio the disturbance growth rates may be either positive or negative, depending of the wavelength $\lambda^{*}$. This differs significantly from the behavior found in the more familiar displacement front first studied by Saffman and Taylor [7]. For that problem, disturbance growth rates do not depend on the disturbance wavelength and are strictly positive or negative depending only on the viscosity ratio and normalized fluid speed.

Neutral stability is given by the condition $\sigma^{*}=0$, yielding an initial front disturbance that neither grows nor decays but instead propagates at constant amplitude with the mean flow. For given values of the two mean velocities, $u_{2}^{*}$ and $U^{*}$, and the normalized wavelength, $\lambda^{*}=2 \pi / \beta^{*}$, there exists some critical value of the viscosity ratio $\mu_{2}^{*}=\mu_{2} / \mu_{1}$ corresponding to this neutral stability condition. To identify these states we use a procedure similar to that used to determine the disturbance growth rates, except that $\sigma^{*}$ is set to zero and $\alpha_{1}^{*}$, $\alpha_{2}^{*}$ and $\mu_{2}^{*}$ are varied to satisfy the three governing algebraic equations.

Conditions of neutral stability are shown in Figure 3 for a dimensionless fluid velocity of $u_{2}^{*}=1.0$ and various values for the front speed $U^{*}$. This value of the fluid velocity is equivalent to $\bar{u}_{1}=\bar{u}_{2}$ and corresponds to the case of no change in temperature and no net sorption at the front, or equivalently to a net sorption and offsetting temperature jump. The parameter on the vertical axis is

$$
\phi=\mu_{2}^{*} u_{2}^{*}=\frac{\mu_{2} \tilde{u}_{2}}{\mu_{1} \tilde{u}_{1}}
$$

By equation (12), $\phi$ is equal to the ratio of the pressure gradient ahead of the front to that behind. In previous analyses of interface stability in porous materials, this ratio of the pressure gradients has been the controlling parameter in determining stable and unstable conditions - not only in the classic Saffman-Taylor problem [7] but also in more complex problems involving phase change [9] and multicomponent flows [10]. In these problems the interface was stable for pressure gradient ratios less than unity and, conversely, unstable for ratios greater than unity. We see in Figure 3 that the pressure gradient criterion also applies to exchange front stability, but only for disturbances of very short wavelength. Disturbances 


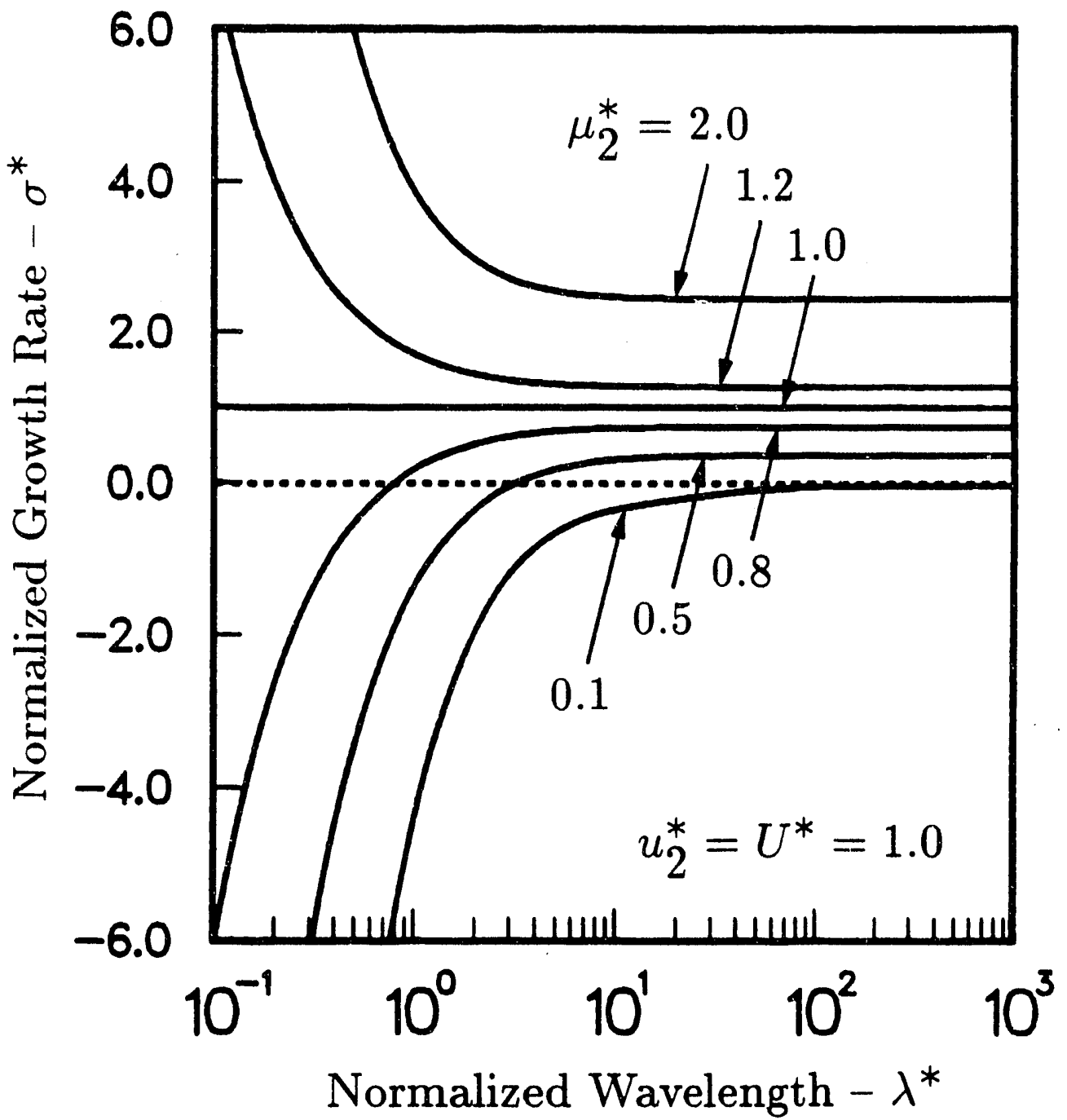

Figure 2. Disturbance growth rates for $u_{2}^{*}=\tilde{u}_{2} / \tilde{u}_{1}=1.0$ and $U^{*}=\epsilon U / \tilde{u}_{1}=1.0$. Growth rates are strongly influenced by the viscosity ratio $\mu_{2}^{*}=\mu_{2} / \mu_{1}$ and normalized wavelength $\lambda^{*}=\lambda / \ell$. For small values of the viscosity ratio, growth rates may be either positive or negative depending on the wavelength. Positive growth rates indicate an unstable front, while negative rates indicate stability. The condition $\sigma^{*}=0$ defines neutral stability. 


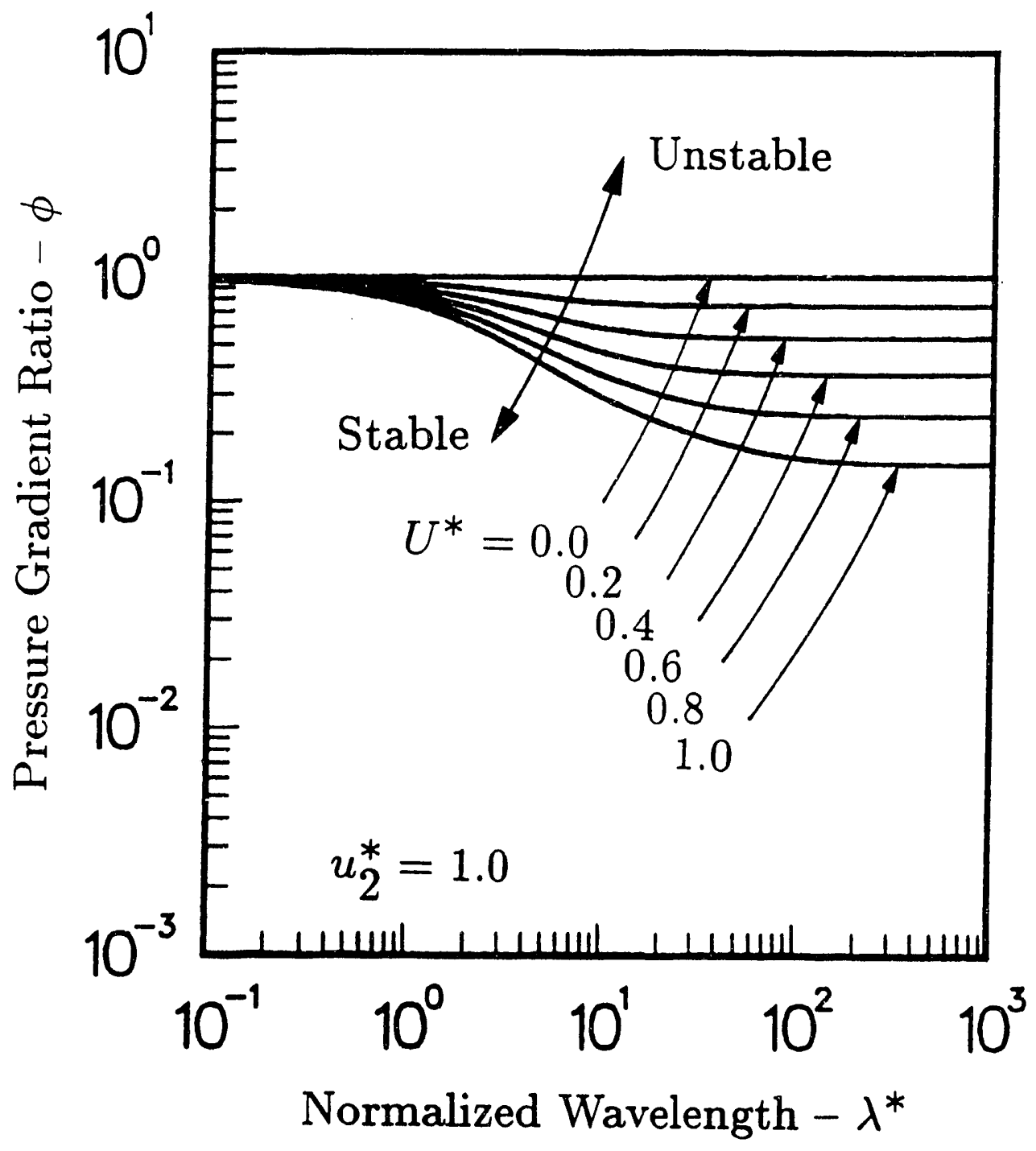

Figure 3. Neutral stability curves for a normalized fluid velocity $u_{2}^{*}=\tilde{u}_{2} / \tilde{u}_{1}=1.0$. The parameter $\phi=\mu_{2}^{*} u_{2}^{*}$ is the ratio of the pressure gradient ahead of the front to that behind; the condition $\phi=1$ is the Saffman-Taylor criterion for neutral stability of a displacement front. Increasing the normalized front velocity $U^{*}$ destabilizes the front at long wavelengths due to fluid compressibility. 
of longer wavelengths may be unstable even when the ratio of pressure gradients favors stability.

The destabilization of long wavelength disturbances is a consequence of fluid compressibility and does not result from the disparity between the exchange front and fluid velocities. On the contrary, Figure 3 shows that the Saffman-Taylor result (a horizontal line at unity) is recovered in the limit $U^{*}=0$ when the velocity of the front is negligible compared to that of the fluid. Moreover, destabilization is greatest for the special case $U^{*}=1$ when the fluid and front velocities are equal, as in the Saffman-Taylor problem. The mathematical origin for this is evident when comparing equations (31) and (32); the dominant contribution from compressibility in equation (31) is multiplied by the front speed $U^{*}$. For this reason both equations (31) and (32) degenerate to $\mu_{2}^{*} u_{2}^{*}=1$ for the neutral stability condition, provided that $U^{*}=0$. Further reviewing equations (21) and (31), we see that the neutral stability curves for $U^{*} \neq 0$ are shifted from the Saffman-Taylor condition by terms arising from the product of the mean density gradient $d \bar{\rho}_{i} / d z$ and front perturbation $z^{\prime}$, not by the density perturbations $\rho_{i}^{\prime}$ resulting from the pressure perturbation $p_{i}^{\prime}$. A physical explanation for the destabilizing influence of fluid compressibility is that a fluid finger growing in a region of falling pressure must accelerate as the fluid expands. This reinforces the instability that normally occurs when the pressure gradient ahead of the front is steeper than that behind.

When exchange at the front is accompanied by a net absorption of gas, the fluid velocity ahead of the front must be smaller than that behind if the temperatures $T_{2}$ and $T_{1}$ are equal. Similarly, in the absence of net sorption a temperature jump such that $T_{1}>T_{2}$ produces a density contrast across the front and again gives a fluid velocity ahead of the front that is smaller than that behind. This is given by $u_{2}^{*}<1$, and in the limit $u_{2}^{*} \rightarrow 0$ the exchange front degenerates to a simple absorption wave. Neutral stability curves for $u_{2}^{*}=0.2$ are shown in Figure 4. Comparing these results with those in Figure 3 shows that reducing the normalized fluid velocity shifts the neutral stability curves still further from the Saffman-Taylor result. However, since we are plotting the critical pressure gradient ratio, not the critical viscosity ratio, this shift from $\mu_{2}^{*} u_{2}^{*}=1$ does not necessarily imply a destabilizing influence. If we instead plotted the critical viscosity ratio, all curves in Figure 4 would be raised by a factor of $1 / u_{2}^{*}=5$. For a fixed viscosity ratio we then see that reducing $u_{2}^{*}$ stabilizes the exchange front at short wavelengths. At longer wavelengths, smaller $u_{2}^{*}$ stabilizes the front at small front velocities, but destabilizes the front as $U^{*}$ increases. 


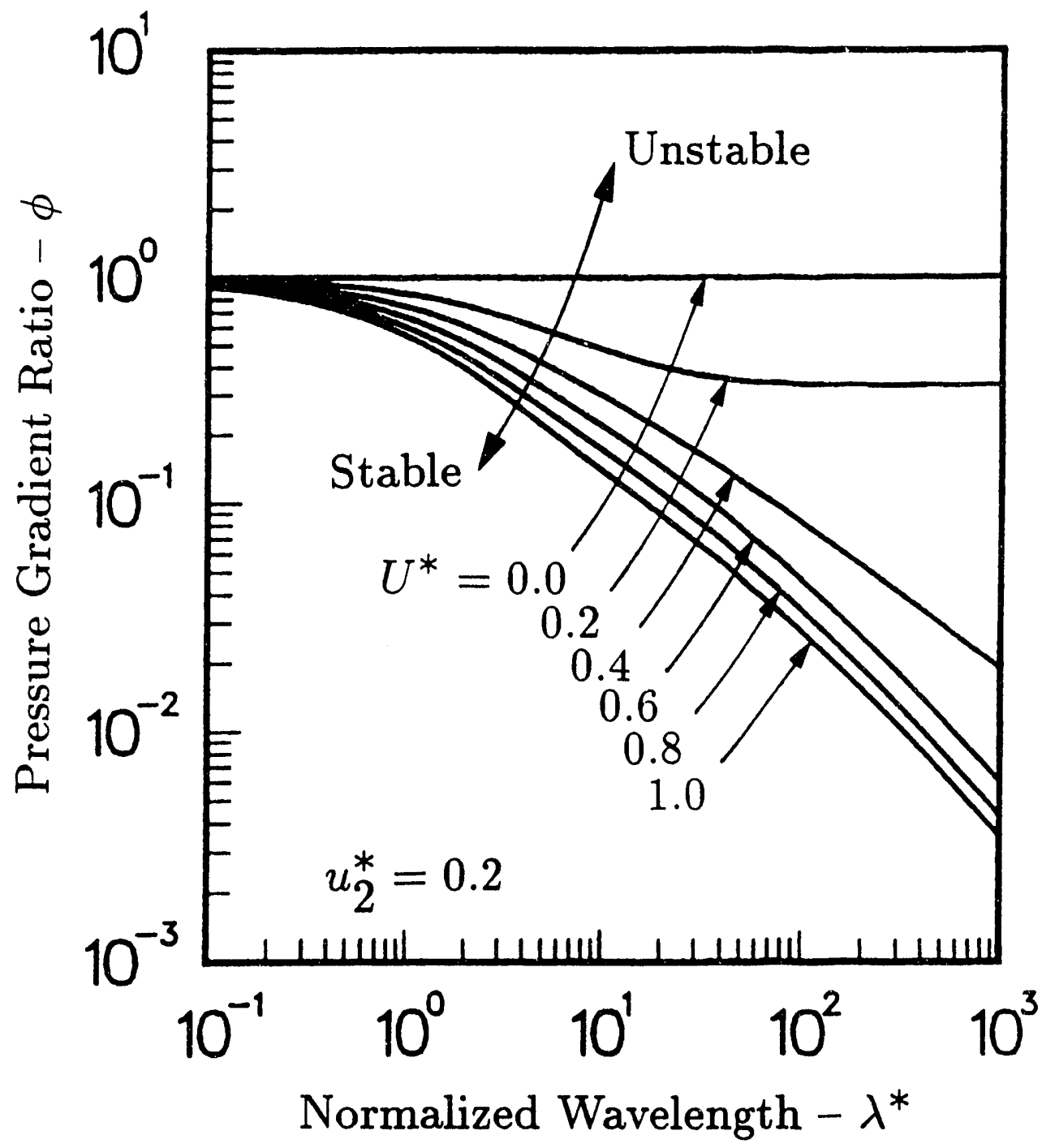

Figure 4. Neutral stability curves for $u_{2}^{*}=\tilde{u}_{2} / \bar{u}_{1}=0.2$. Values of $u_{2}^{*}$ less than unity correspond to a temperature rise or net absorption of gas at the exchange front. For a fixed pressure gradient ratio, absorption always destabilizes the front. For a fixed viscosity ratio, however, absorption stabilizes short wavelength disturbances; longer wavelengths may be destabilized if the front velocity $U^{*}$ is large. 


\section{SAMPLE CALCULATIONS}

One unusual use for exchange chromatography is in the storage and purification of hydrogen isotopes. In this application hydrogen is stored in the column as a metal hydride [11], commonly uranium, palladium or lanthanum-nickel alloys. Within the hydride, hydrogen atoms reside between metal atoms at atomic hydrogen-to-metal ratios of order unity. Because of this, very high storage densities can be obtained, often without large pressures. For example, at ambient temperature and an equilibrium pressure of only one atmosphere, the protium-to-metal ratio is about $s=0.7$ for palladium and about $s=3.0$ for uranium. These correspond to protium densities of 70 and $46 \mathrm{~kg} / \mathrm{m}^{3}$, respectively; the gas-phase density of protium at these conditions is only $0.08 \mathrm{~kg} / \mathrm{m}^{3}$. Low pressure storage of hydrogen is important because high pressure hydrogen embrittles many ferrous alloys.

Hydrogen isotopes stored in a porous hydride column can be recovered either by heating the column or through elution by another isotope. When a column containing one isotope is flushed by an influent stream of a second, an exchange front propagates along the column as the isotope initially on the column is displaced via exchange between the gas and solid phases. Until the leading edge of the exchange front reaches the column exit, the effluent stream will contain only that isotope initially on the column. The exchange kinetics for metal hydrides are relatively fast [12], and hydrogen recovery by elution is efficient provided that the hydride surfaces are clean and that the exchange front remains relatively planar. If the exchange front deviates significantly from the planar geometry, the front will emerge from the column progressively, resulting in a prolonged period of mixed isotopes in the effluent stream and poor column performance.

In addition to storage applications, porous hydride columns can be used for isotope separation. The three isotopes of hydrogen exhibit distinct equilibrium constants [13], so the saturation of a column with mixed isotopes produces a column composition slightly enriched in the more favored species. Repeated cycles of saturation and elution therefore can be used to isolate a single isotope from a mixture. Again, high efficiency for this process requires a stable planar exchange front.

For a sample problem we will consider a column of palladium hydride powder at a porosity of $\epsilon=0.7$. The column is initially saturated with only one of the three isotopes and is eluted by an undiluted influent stream of a second. We will compute the normalized disturbance growth rates for each of the six non-trivial isotope pairs at an exchange front pressure of $\tilde{p}=6.8 \mathrm{MPa}(1000 \mathrm{psi})$. An undiluted influent stream is given by $\psi=0$, and the effective solid-phase hydrogen densities are then simply $\rho_{i}^{\psi}=\rho_{i}^{s}$. The diatomic hydrogen density in the solid phase is $\rho_{i}^{s}=0.05 s_{i} \mathrm{~mol} / \mathrm{cm}^{3}$, where the hydrogen-to-metal ratio $s_{i}$ is given by

$$
\ln \left(\frac{\bar{p}_{i}}{p_{a}}\right)=d_{j}-\frac{\left(e_{j}-f_{j} s_{i}\right)}{T_{i}}+g_{j} T_{i}
$$


Here the reference pressure $p_{a}=101.3 \mathrm{kPa}$ is one atmosphere, and the constants $d_{j}, e_{j}, f_{j}$ and $g_{j}$ for each of the isotopes $j=\mathrm{H}, \mathrm{D}$ or $\mathrm{T}$ are obtained by fitting measured pressurecomposition isotherms over a range of temperatures [14]. The metal atom density of palladium hydride is $\rho_{s}=0.10 \mathrm{~mol} / \mathrm{cm}^{3}$, and its specific heat (excluding the hydrogen) is $c_{v}=25.5 \mathrm{~J} / \mathrm{mol}$. Specific heats of the gas-phase hydrogen are $c_{p, i}=29.1 \mathrm{~J} / \mathrm{mol}$, and those of the hydrogen in the solid are $c_{v, i}^{s}=33.4 \mathrm{~J} / \mathrm{mol}[15]$. Fluid viscosities for all isotopes are assumed to be proportional to the square-root of the product of the temperature and atomic mass. The palladium hydride enthalpies of formation are $h_{j}=-3.90,-3.54$ and $-3.33 \mathrm{~kJ} / \mathrm{mol}$ for protium, deuterium and tritium [16].

Using the material characteristics above, the normalized fluid velocities and viscosity ratio can be computed for a given isotope pair. We calculate these parameters by an iterative technique to find $s_{1}, T_{1}, u_{2} / \epsilon U$ and $u_{1} / \epsilon U$ given the exchange front pressure $\bar{p}$ and initial column temperature $T_{2}$. The procedure is to first calculate the initial hydrogen-to-metal ratio $s_{2}$ from $\tilde{p}$ and $T_{2}$. Using this and a guessed value for $s_{1}$, the solid-phase hydrogen densities $\rho_{i}^{s}$ are determined and a trial value for $T_{1}$ is calculated from equation (8b). A trial value for $\bar{p}_{1}$ is then calculated using $s_{1}, T_{1}$ and equation (34), and this pressure is compared with the specified pressure at the front. Again the ZEROIN routine is used to select a new value for $s_{1}$ based on the discrepancy in the pressures, and the procedure is repeated until $\bar{p}_{1}$ and $\tilde{p}$ converge. Once $T_{1}$ is determined the viscosity ratio $\mu_{2}^{*}=\mu_{2} / \mu_{1}$ is computed, and the continuity matching conditions (14) and the equation-of-state are used to calculate $\bar{u}_{1} / \epsilon U$ and $\bar{u}_{2} / \epsilon U$. From these the dimensionless parameters $u_{2}^{*}=\bar{u}_{2} / \bar{u}_{1}$ and $U^{*}=\epsilon U / \bar{u}_{1}$ are determined. The resulting dimensionless parameters are shown in Table 1 for each of the six isotope pairs; H-D, H-T, D-H, D-T, T-H and T-D. In each case the first of the pair designates the hydrogen isotope of the influent stream; the second designates that initially in the column.

\begin{tabular}{|l|l|l|l|l|l|l|}
\cline { 2 - 6 } \multicolumn{1}{c|}{} & $\mathrm{H}-\mathrm{D}$ & $\mathrm{H}-\mathrm{T}$ & $\mathrm{D}-\mathrm{H}$ & $\mathrm{D}-\mathrm{T}$ & $\mathrm{T}-\mathrm{H}$ & $\mathrm{T}-\mathrm{D}$ \\
\hline$T_{2} / T_{1}$ & 0.86 & 0.80 & 1.23 & 0.91 & 1.40 & 1.11 \\
$\mu_{2}^{*}$ & 1.31 & 1.55 & 0.78 & 1.17 & 0.68 & 0.86 \\
$u_{2}^{*}$ & 0.88 & 0.83 & 1.19 & 0.91 & 1.32 & 1.10 \\
$U^{*}$ & 0.13 & 0.12 & 0.16 & 0.14 & 0.18 & 0.16 \\
$\mu_{2}^{*} u_{2}^{*}$ & 1.14 & 1.28 & 0.93 & 1.07 & 0.90 & 0.95 \\
\hline
\end{tabular}

Table 1. Calculated parameters for hydrogen isotope exchange in a packed-powder palladium column. These values depend only on the exchange front pressure, initial temperature and bed porosity; they do not depend on the magnitude of the fluid or front velocities. The parameters shown are for the conditions $\tilde{p}=6.8 \mathrm{MPa}(1000 \mathrm{psi}), T_{2}=20 \mathrm{C}$ and $\epsilon=0.7$. 
Using the values in Table 1 , the normalized growth rate $\sigma^{*}$ and spatial decay rates $\alpha_{i}^{*}$ can be computed by solving the coupled algebraic system given by equations (29), (30) and (31). The resulting normalized disturbance growth rate for each of the six isotope pairs is shown in Figure 5. We see that the exchange front has positive growth rates at all wavelengths for the H-D, H-T and D-T isotope pairs. Thus the exchange front is strictly unstabie in all cases where the displacing isotope has a lower atomic mass than that initially on the column. Similarly, those pairs in which the displacing isotope has the higher mass yield negative disturbance growth rates and stable fronts, but only at short wavelengths. Though not readily apparent in the Figure 5, the growth rates for these cases pass through $\sigma^{*}=0$ at normalized wavelengths of $\lambda^{*}=1.8,2.5$ and 1.2 for the D-H, T-H, and T-D isotope pairs. At still longer wavelengths these pairs also exhibit an unstable exchange front.

Despite the fact that isotope exchange fronts are in many cases unstable, the dimensional growth rate may be quite small. For example, at a fluid velocity of $\tilde{u}_{1}=5 \mathrm{~mm} / \mathrm{s}$, influent viscosity $\mu_{1}=0.9 \times 10^{-5} \mathrm{~Pa}$-s and column permeability of $k=10^{-14} \mathrm{~m}^{2}$ the characteristic length for our sample problem is $\ell=1.5 \mathrm{~m}$ and the characteristic time is $\epsilon \ell / \tilde{u}_{1}=210 \mathrm{~s}$. At a wavelength of $10 \mathrm{~cm}$, corresponding to $\lambda^{*}=0.067$, the normalized growth rate for the $\mathrm{H}-\mathrm{D}$ pair is $\sigma^{*}=0.88$ giving a dimensional growth rate of $0.0042 \mathrm{~s}^{-1}$. Thus for exponential growth, the time for a front disturbance to double in amplitude is $170 \mathrm{~s}$. The doubling time increases with increasing wavelength, and in the limit $\lambda^{*} \rightarrow \infty$ the normalized growth rate is only $\sigma^{*}=0.042$. This gives a dimensional growth rate of $0.0002 \mathrm{~s}^{-1}$ and an amplitude doubling time of nearly $3500 \mathrm{~s}$ for the H-D pair. Because these growth rates are fairly small, hydrogen exchange columns may perform well even for isotope pairs yielding a fundamentally unstable exchange front, provided that the column is not too long.

To examine the effect of column length on disturbance growth, we consider an idealized case in which the exchange front velocity is constant along the column. In this case the time to breakthrough is $t=L / \bar{U}$, where $L$ is the column length. Although the normalized front velocity must fall with the exchange front pressure since $\tilde{u}_{1} \propto 1 / \bar{p}$, the disturbance growth rate is roughly proportional to the normalized front velocity, so we can further assume that the ratio $\sigma^{*} / U^{*}$ is nearly constant as the front progresses along the column. Using equation (27c), the exponent of the disturbance growth exponential therefore can be written

$$
\sigma t \approx \frac{\sigma^{*} L}{U^{*} \ell}
$$

and to double the disturbance amplitude then requires a column length of $L=0.7 U^{*} \ell / \sigma^{*}$. For the H-D pair in our sample problem this length is $15 \mathrm{~cm}$ at a normalized growth rate of $\sigma^{*}=0.88$ and $3.2 \mathrm{~m}$ at $\sigma^{*}=0.042$. 


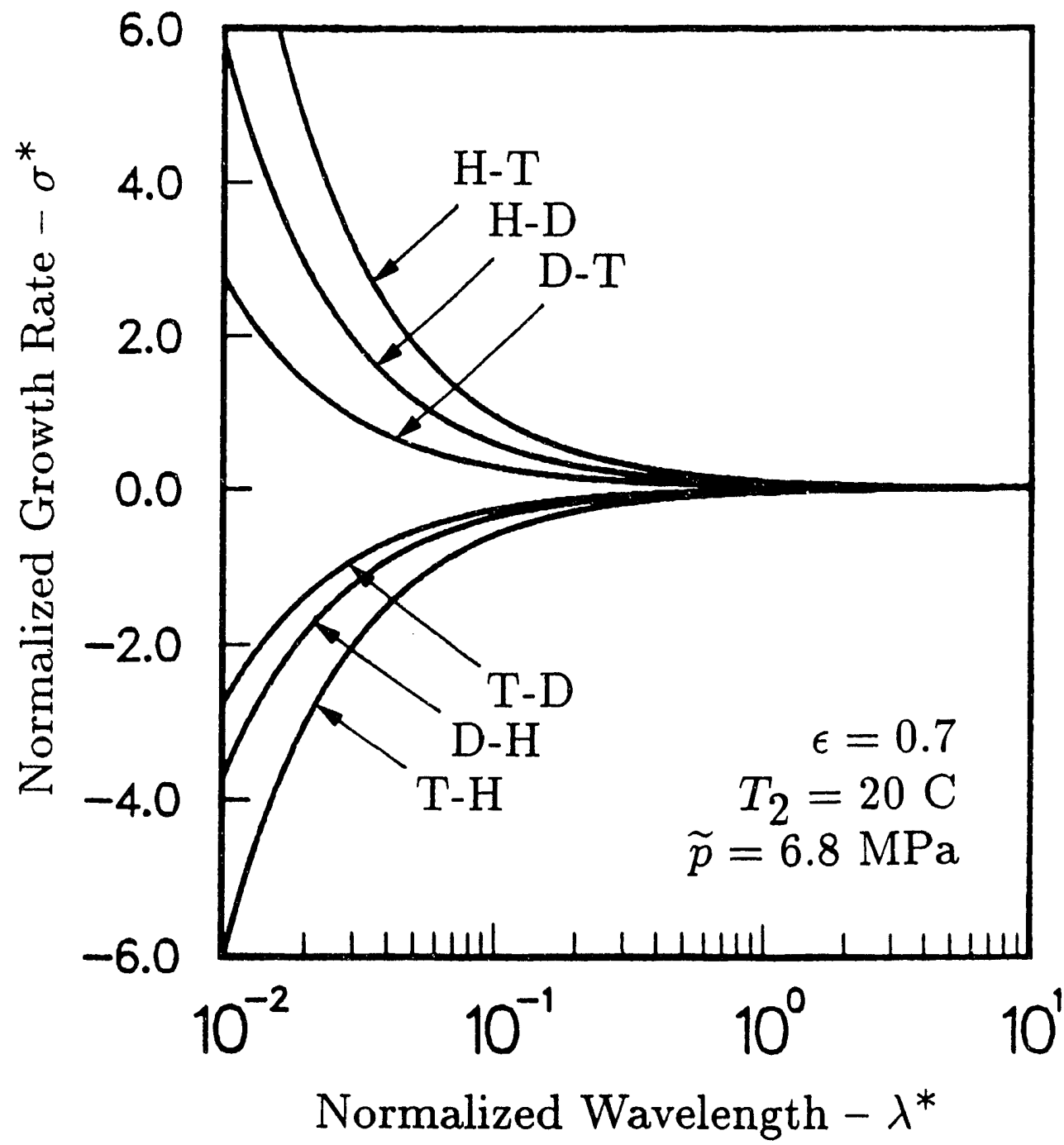

Figure 5. Disturbance growth rates for exchange of hydrogen isotope pairs. Pairs in which the displacing isotope has the lower atomic mass possess unstable exchange fronts at all wavelengths. When the mass of the displacing isotope is the higher of the pair, exchange fronts are stable at short wavelengths. The asymptotic values of the normalized growth rates at very long wavelengths are $0.033,0.042,0.015,0.029,0.014$ and 0.018 for H-D, H-T, D-H, D-T, T-H and T-D, respectively. 


\section{SUMMARY}

Our stability analysis yields three coupled non-linear algebraic equations relating the temporal growth rate and spatial decay of a small disturbance to a planar exchange front. From these equations the growth rate can be computer as a function of the wavelength, viscosity ratio $\mu_{2} / \mu_{1}$, and the two normalized velocities $\bar{U} / \bar{u}_{1}$ and $\bar{u}_{2} / \bar{u}_{1}$. The normalized front velocity $\bar{U} / \tilde{u}_{1}$ and fluid velocity ratio $\tilde{u}_{2} / \tilde{u}_{1}$ depend only on the local gas density, solid storage capacity, and the equilibrium partition of species between the gas and solid phases. No additional parameters are needed to account for compressibility because density gradients are directly related to pressure gradients for an ideal gas. As in the Saffman-Taylor problem for a displacement front, the growth rate of exchange front disturbances increases with increasing viscosity ratio and decreasing wavelength. Here the growth rate additionally increases with larger normalized front velocities and larger fluid velocity ratios.

The conditions of neutral stability are also determined. We find that exchange fronts are generally stabilized when the fluid velocity ahead of the front is smaller than that behind, $\bar{u}_{2} / \tilde{u}_{1}<1$, corresponding to a temperature rise or net loss of gas at the exchange front. This is due to the lower fluid velocity and accompanying decreased magnitude of the pressure gradient ahead of the front, which is always favorable to front stability. However, the stabilizing influence of sorptive losses is sometimes offset by the contribution of compressibility, which tends to destabilize the front due to the expansion and accompanying acceleration of gases ahead of the front. Plots of the neutral stability conditions are presented for a wide range of wavelengths, normalized front velocities and fluid velocity ratios.

Finally, sample calculations for isotope exchange in a palladium hydride column show that the hydrogen exchange front is unstable at all wavelengths when the displacing isotope has a lower atomic mass than the mass of the isotope initially on the column. When the mass of the displacing isotope is the higher of the two, exchange fronts are stable at short wavelengths, but at longer wavelengths these isotope pairs also yield unstable fronts. Disturbance growth rates for our sample problem are generally small, however, and we would expect only moderate front deformation for columns less than 10 to $100 \mathrm{~cm}$ in length. 


\section{REFERENCES}

1. W. H. Flank, Ed., Adsorption and Ion Exchange with Synthetic Zeolites, ACS Symposium Series, Vol. 135, American Chemical Society, Washington, D. C., 1980.

2. H. Purnell, Gas Chromatography, John Wiley and Sons, New York, NY, 1962.

3. T. B. Drew and J. W. Hoopes, Jr., Eds., Advances in Chemical Engineering, Vol. II, 147-209, Academic Press, New York, NY, 1958.

4. J. B. Rosen, "Kinetics of a Fixed Bed System for Solid Diffusion into Spherical Particles," J. Chem. Phys., Vol. 20, No. 3, 1952.

5. S. Hill, "Channelling in Packed Columns," Chem. Eng. Sci., Vol. I, No. 6, 247-253, 1952

6. R. L. Chuoke, P. van Meurs, and C. van der Poel, "The Instability of Slow, Immiscible, Viscous Liquid-liquid Displacements in Permeable Media, AIME Pet. Trans., Vol. 216, 188-194, 1958.

7. P. G. Saffman and G. I. Taylor, "The Penetration of a Fluid into a Porous Medium of Hele-Shaw Cell Containing a More Viscous Fluid," Proc. R. Soc. London, Ser. A, Vol. 245, 312-329, 1958.

8. G. E. Forsythe, M. A. Malcom and C. B. Moler, Computer Methods for Mathematical Computations, Prentice-Hall, Englewood Cliffs, NJ, 1977.

9. C. A. Miller, "Stability of Moving Surfaces in Fluid Systems with Heat and Mass Transport," AIChE J., Vol.21, No. 31, 474-479, 1975.

10. J. M. Laude and F. A. Morrison, Jr., "Stability of Flow From a Nuclear Cavity," ASME J. Fluids Eng., Vol. 101, 335-340, 1979.

11. W. M. Mueller, J. P. Blackledge and G. G. Libowitz, Eds., Metal Hydrides, Academic Press, New York, NY, 1968.

12. G. Sicking, P. Albers and E. Magomedbekov, "Hydrogen Isotope Exchange and Separation in Gas-Solid Phase Systems," J. I,ess-Common Metals, Vol 89, 373-391, 1983.

13. G. L. Powell and R. Lasser, "Solubility of Hydrogen, Deuterium and Tritium in Palladium Hydride," Internal Report Y-2395, Oak Ridge Y-12 Plant, Oak Ridge, TN, 1988.

14. F. A. Lewis, The Palladium Hydrogen System, Academic Press, New York, NY, 1967.

15. P. Mitacek, Jr., and J. G. Aston, "The Thermodynamic Properties of Pure Palladium and its Alloys with Hydrogen Between 30 and $300 \mathrm{~K}$," J. Am. Chem. Soc., Vol 85, 137-141, 1963.

16. R. Lasser and K. H. Klatt, "Solubility of Hydrogen Isotopes in Palladium," Phys. Rev. B, Vol 28, No. 2, 1983. 
UNLIMITED RELEASE

INITIAL DISTRIBUTION

EG\&G Mound Applied Technologies

Attn: D. S. West, SW-239

P. O. Box 3000

Miamisburg, $\mathrm{OH}$ 45342-3000

Los Alamos National Laboratory

Attn: J. M. Bunch, MS G780

P. O. Box 1663

Los Alamos, NM 87545

Los Alamos National Laboratory

Attn: W. R. Doty, MS G780

P. O. Box 1663

Los Alamos, NM 87545

Los Alamos National Laboratory

Attn: I. E. Lindstrom, MS G780

P. O. Box 1663

Los Alamos, NM 87545

Martin Marietta Energy Systems, Inc.

Oak Ridge Y-12 Plant

Attn: G. L. Powell

P. O. Box Y

Oak Ridge, TN 37831

S-CUBED / Maxwell Labs

Attn: R. H. Nilson (10)

P.O. Box 1620

La Jolla, CA 92038-1620

Westinghouse Savannah River Co.

Attn: M. W. Lee, 773-A

Savannah River Site Aiken, SC 29808

Westinghouse Savannah River Co.

Attn: A. F. Riechman, 773-A

Savannah River Site Aiken, SC 29808
1512

8000

8341

8341

8400

8441

8446

8446

8446

8446

8446

8446

8700

8701

8713

8715

8715

8716

8741

8744

8744

8744

8745

8535

8535

7141

8

8535-2
M. R. Baer

J. C. Crawford

Attn: D. L. Crawford, 1900

E. E. Ives, 5200

J. B. Wright, 5300

M. E. John, 8100

L. A. West, 8600

W. G. Wolfer

D. C. Chrzan

L. A. Hiles

H. H. Hirano

P. D. Gildea

K. R. Blumberg

B. L. Haroldsen

C. A. Lajeunesse

M. C. Stoddard

A. J. West

R. C. Wayne

Attn: C. W. Robinson, 8702

R. J. Kee, 8745

D. L. Lindner

B. E. Mills

G. J. Thomas

S. E. Guthrie

J. M. Hruby

J. C. Keilman

C. M. Hartwig

S. K. Griffiths (10)

B. A. Meyer

A. E. Lutz

Publications for OSTI (10)

Publications/Technical Library Processes, 7141

Technical Library Processes

Department (3) 


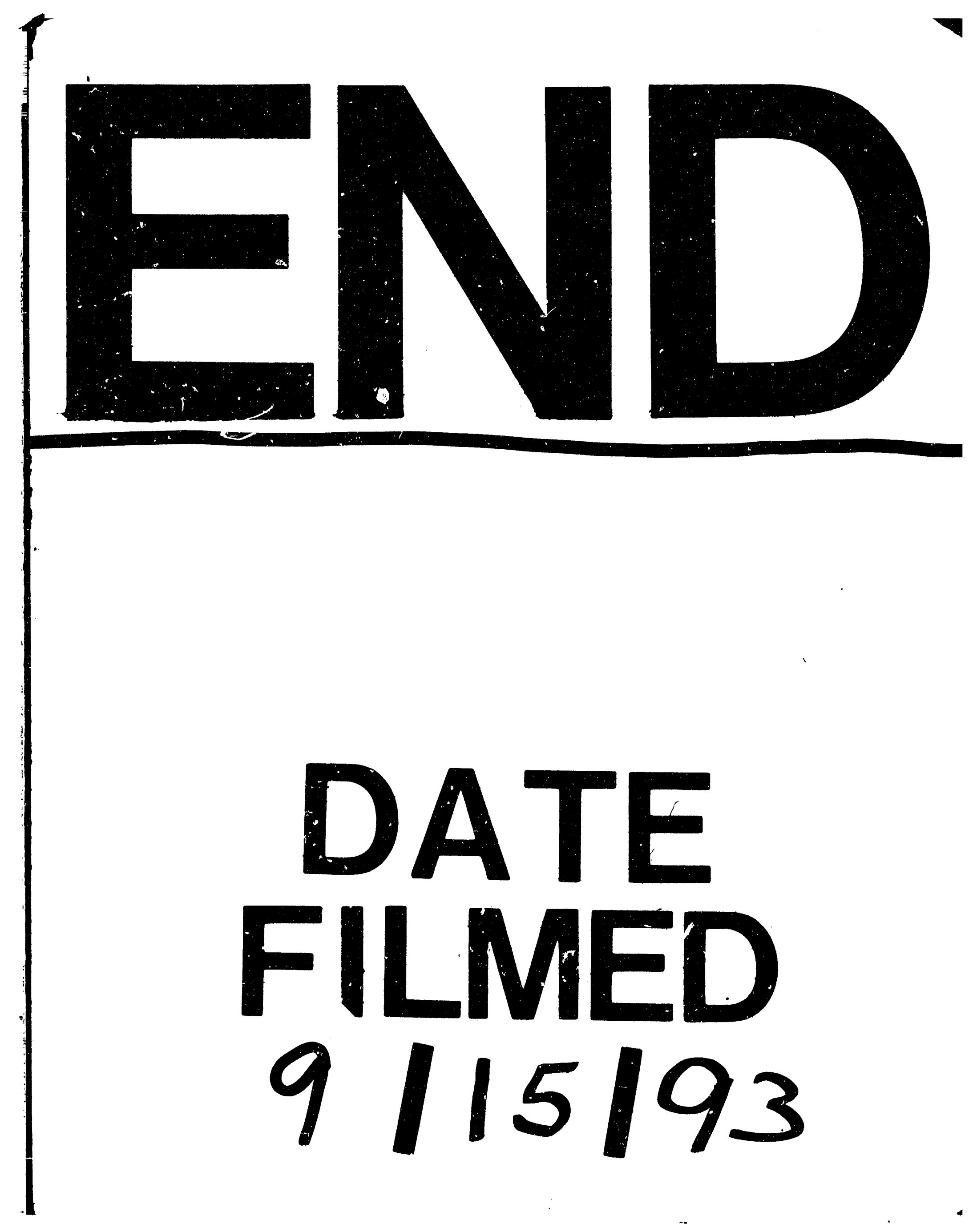


